\title{
Cervical cancer screening program in Thimphu, Bhutan: population coverage and characteristics associated with screening attendance
}

\author{
lacopo Baussano ${ }^{1}$, Ugyen Tshomo², Gary M Clifford', Vanessa Tenet ${ }^{1}$, Tshokey Tshokey ${ }^{3}$ and Silvia Franceschi*
}

\begin{abstract}
Background: Bhutan has been engaged in good-quality cytology-based cervical screening since 2000 and has vaccinated $>90 \%$ girls against human papillomavirus (HPV) since 2010. We explored the characteristics associated with lack of previous screening and screening coverage in women age $\geq 25$ years.

Methods: Women were invited at home or during their attendance at 2 outpatient clinics, in the capital, Thimphu, and nearby Lungthenphu. Age-adjusted odds ratios for lack of previous screening by selected characteristics were computed among 1,620 participating women. In Thimphu an invitation registry allowed to estimate screening history not only among participating women but also among additional 500 women who did not accept to join our study.

Results: Among women who had a Pap smear, lack of previous screening was associated with age $<35$ or $\geq 45$ years. It was also associated with some occupations; being single, or widowed/separated; and presence of HPV infection. Multiparity and use of contraceptive methods were associated with having been screened. In women invited at home in Thimphu screening history substantially differed by participation. Past screening attendance was $59 \%$ among women recruited in the 2 clinics, 53\% in women who were invited from home and accepted the invitation, but only $25 \%$ in those who refused it. Based on all women recruited from home the estimate of population-based coverage in Thimphu is 34\% (95\% Cl: 31-37).

Conclusions: Transition from an opportunistic screening to an all-reaching population-based screening is yet to be achieved in Bhutan, even in the capital. Better ways to target never-screened women are needed.
\end{abstract}

Keywords: Screening, Cervical cancer, Bhutan

\section{Background}

Cervical cancer represents the most common cancer among women in Bhutan, with a world age-standardized incidence rate of 13 cases per 100,000 person years [1]. Cervical cancer incidence is likely to be underestimated on account of the incomplete establishment of the national cancer registry. The age-standardized prevalence of human papillomavirus (HPV) infection in the general female population in Bhutan is 26\%, ranging from 33\% below age 25 years and $19 \%$ at age 45 and above [2]. It is one of the most elevated HPV prevalence among Asian

\footnotetext{
* Correspondence: franceschi@iarc.fr

'International Agency for Research on Cancer, 150 cours Albert Thomas, 69372 Lyon Cedex 08, France

Full list of author information is available at the end of the article
}

countries [3-9]. It is therefore very appropriate that the country (634,982 inhabitants) has seriously engaged in cervical cancer prevention [10]. Bhutan was the first low/middle-income country to start in 2010 a nationwide school-based program of vaccination against HPV and has achieved vaccination-coverage of $92 \%$ among 12 to 18 year old girls [11].

Bhutan has also been on the front line in the secondary prevention of cervical cancer: a national cytologybased screening program, including quality control of Pap smear findings, and systematic recall of screeningpositive women for colposcopy and, if necessary, biopsies and treatment, was launched in 2000 [10]. No active call/recall system is in place but a Pap smear is recommended every three years to women aged 20-60 years 
and it is provided free of cost by trained female health assistants, nurses, and medical doctors in national referral hospitals and district hospitals through maternal and child health clinics. Although Pap smear campaigns are occasionally conducted in rural and high-altitude areas, the majority of screening activity is concentrated in the district of the capital, Thimphu, which hosts approximately one sixth of the Bhutanese population. The only information on the performance of cervical cancer screening in Bhutan is a recent publication that shows poor knowledge of and low participation in cervical cancer screening among female university graduates, mainly below age 25 years [10].

The aims of the present study were to provide an estimate of screening coverage in Thimphu and explore the characteristics associated with lack of previous screening among women age 25 or older. The evaluation of screening was part of a large HPV survey carried out by the Jigme Dorji Wangchuck National Referral Hospital (JDWNRH), Thimphu, in collaboration with the Ministry of Health of Bhutan, and the International Agency for Research on Cancer (IARC) to monitor future impact of HPV vaccination and the transition from cytology to HPV testing in cervical cancer screening program.

\section{Methods}

Between December 2011 and October 2012, 2,505 sexually active women age 18-69 years underwent conventional cytology and HPV testing in JDWNRH, Thimphu, and in the Hospital of Lungthenphu, a satellite town in the district of Thimphu, to provide baseline HPV prevalence prior to HPV vaccination. Study procedures have been described elsewhere [2]. In brief, women in both study areas were recruited in two ways (Figure 1). Residents of pre-defined areas surrounding the two hospitals were visited at home by social workers and invited to join the study (participation: $31 \%$ in Thimphu and $45 \%$ in Lungthenphu). Women consulting outpatient clinics in the two hospitals for cervical screening (mainly antenatal care or family planning clinics) were invited to join the study (participation $\sim 100 \%$ ). Following signature of an informed consent form, a structured questionnaire including information on screening history and other socio-demographic and lifestyle characteristics was administered to study participants. Testing for $44 \mathrm{HPV}$ types was made using a general primer GP5+/6+ -mediated PCR and reverse-line blot hybridization [12].

To investigate screening participation in recommended age groups [13], the present report focused on 1,620 women aged 25 years or above (median age $=33$, inter-quartile range: 28-43) [2]. Among them, 1,003 were seen at the JDWNRH and 617 at the Lungthenphu Hospital. In Thimphu only, an invitation registry including age, marital status, and screening history was kept and allowed the additional evaluation of all 500 women 25 years or above who had been invited at home in Thimphu but did not eventually participate in the study (Figure 1).

Odds ratios (OR) for lack of previous screening and corresponding 95\% confidence intervals (CI) were computed by unconditional logistic regression adjusted for 5-year age groups. Additional adjustment for type of recruitment did not materially modify any findings. $\chi^{2}$ test for trend were computed by considering categories as continuous variables. Effect modification was tested using the log-likelihood ratio test.

The present study had the approval of both the Bhutan Research Ethics Board of Health and the IARC Ethics Committee.

\section{Results}

Overall, 661 (40.8\%) women who took part in our study had never been screened before. Table 1 shows the relationship between screening history and selected women's characteristics among study participants. No significant difference was found between the two study areas. The lowest fraction of never-screened women was found in the age group 35-44 years (28.0\%). Lack of previous screening was more frequent in women below age 35 years and those age 45 or above (OR versus age 35$44=2.3$; 95\% CI:1.8-3.0; and 1.7; 95\% CI: 1.2-2.3, respectively). Compared to housewives, the fraction of never-screened women was larger in shopkeepers/saleswomen $(\mathrm{OR}=1.6 ; 95 \% \mathrm{CI}: 1.1-2.3)$ and manual workers/ farmers $(\mathrm{OR}=1.7 ; 95 \% \mathrm{CI}: 1.2-2.4)$. Among ethnic groups, a relative lack of previous screening was found in Lhotsampa (OR vs. Scharchop =1.6; 95\% CI: 1.2-2.1). ORs for lack of previous screening in single and widowed/separated women $v s$. married women were 4.9 (95\% CI: 1.3 17.9), and 1.8 (95\% CI: 1.2 - 2.6), respectively.

The fraction of never-screened women decreased with number of pregnancies $\left(\chi^{2}\right.$ for trend among women who had been pregnant $=9.8, \mathrm{p}=0.002$ ) and it was largest among nulligravidae (OR for 0 vs. 1 child $=1.9$; 95\% CI: 1.2-3.2). Fewer never-screened women were found among current users of any contraceptive method (OR in users of hormonal contraceptives and other methods vs. non users $=0.5,95 \% \mathrm{CI}$ : 0.3-0.6; and 0.4; 95\% CI: $0.3-$ 0.5 , respectively). Finally, lifetime sexual partners (OR for $\geq 2$ vs $1=1.4 ; 95 \% \mathrm{CI}: 1.0-1.9)$ and presence of $\mathrm{HPV}$ infection $(\mathrm{OR}=1.5 ; 95 \% \mathrm{CI}: 1.2-1.9)$ were associated with lack of previous screening (Table 1).

Education level was positively and significantly associated with previous screening attendance among women age 45 or above $\left(\chi^{2}\right.$ for trend $\left.=5.3 \mathrm{p}=0.02\right)$ but not in younger age groups (p-value for heterogeneity $=0.06$ ) (Figure 2a). Among 959 previously screened women, over $70 \%$ in every age group reported a Pap smear 


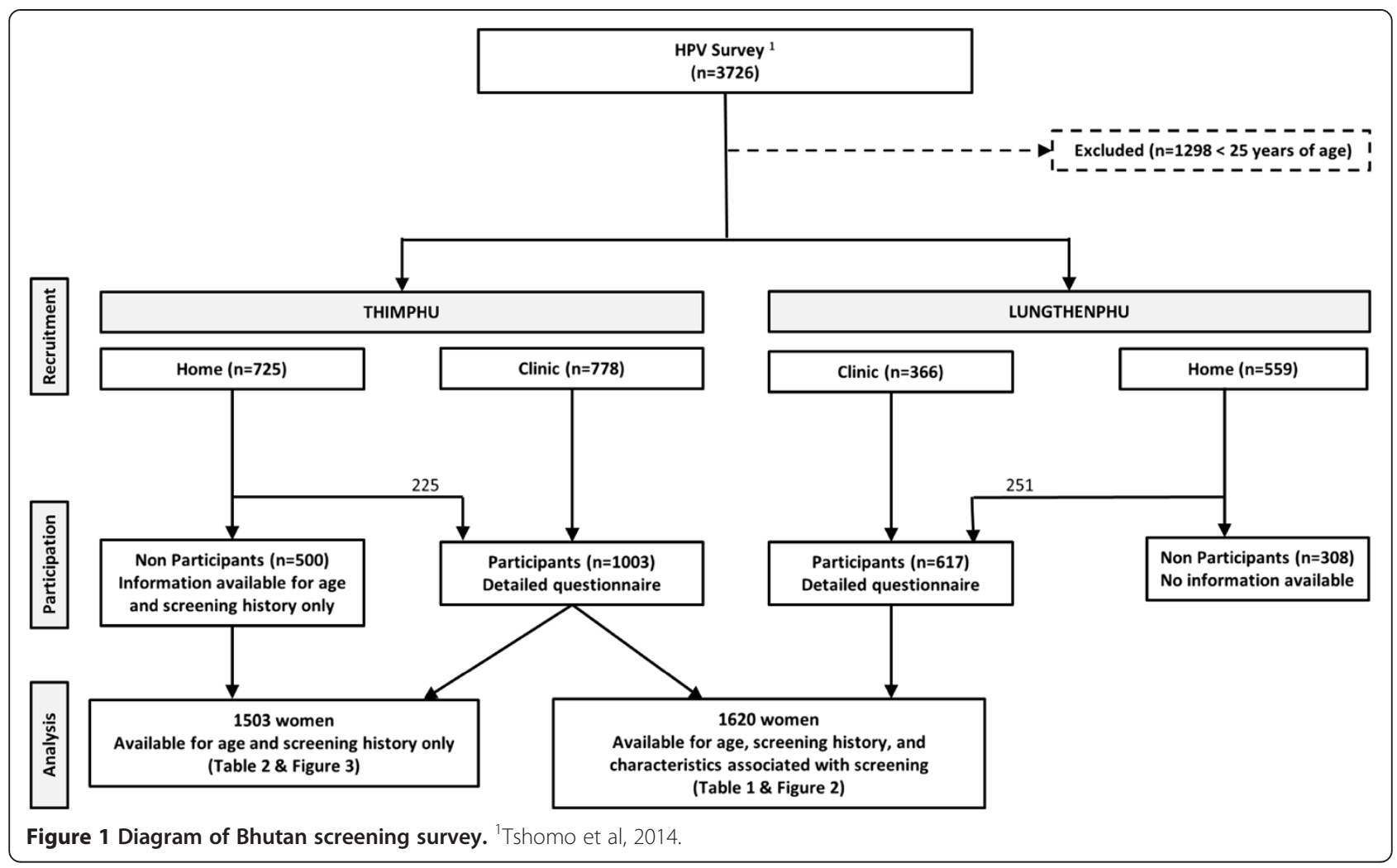

within the last three years and few reported an interval of six years or more (Figure $2 \mathrm{~b}$ ).

Table 2 shows screening history among 1,503 women from Thimphu according to the place in which they were contacted and whether they accepted the invitation. Among women invited from home, the invitation compliance was $31 \%$. It was $28 \%, 36 \%$, and $31 \%$ in women $<35 ; 35-44$, and $\geq 45$, respectively (data not shown). The largest fraction of lack of screening (75\%) was found among women who were contacted at home but did not participate in our study (OR for lack of screening $v s$ women who were contacted at the clinic $=4.3$; 95\% CI: 3.4-5.6). We notice that only 105 (21.9\%) of 479 never-screened women who were invited from home could eventually be screened for the first time in the framework of our study. Past screening attendance in all women contacted at home was $33.9 \%$ (95\% CI: 30.537.3) and we considered it a proxy of population-based coverage (Table 2).

Figure 3 shows that the gap in coverage by place in which women were invited and according to study participation increased with age. Among women age 45 years or older screening coverage among all women who were contacted at home was $23.9 \%$ (95\% CI: 16.9-32.0).

\section{Discussion}

Our study showed for the first time that the cervical cancer screening program in Thimphu reaches approximately one third of women age 25 or older and lack of previous screening was especially frequent among women who refused the invitation to join our study. They, therefore, missed the opportunity to be screened for the first time in the framework of our study.

Among women who took part in our study, previous screening attendance was relatively high (59\%) and better than in the majority of the 16 low/middle-income populations who were formerly studied in methodologically consistent IARC HPV Surveys, i.e., similar recruitment protocol, age distribution, participation level, and location in places in which at least minimum cervical screening expertise and infrastructure were already present [2-9,14-20] (Figure 4). Much lower levels of screening attendance were found in Nepal, China, Pakistan, and Vietnam. Only in Tehran, Iran; Argentina, Concordia; and Lampang, Thailand was the proportion of previously screened women larger than in Bhutan. We notice that the proportions of ever-screened women in previous IARC HPV Surveys were probably affected by a similar participation bias as in the figure from Bhutan.

Among study participants in Thimphu and Lungthenphu, screening history was also significantly affected by several factors. Past screening attendance was more frequently reported by women 35 to 45 years old than by younger or older women. Being married, having had children, and using any type of contraceptive method were also significantly and positively associated with past 
Table 1 Odds ratio and $95 \%$ confidence interval for lack of prior cervical cancer screening by selected women's characteristics, 1,620 women, Thimphu and Lungthenphu, Bhutan

\begin{tabular}{|c|c|c|c|c|}
\hline Variable & Total & N. (\%) of women never screened & Odds ratio $^{a}$ & 95\% confidence interval \\
\hline All women & 1620 & $661(40.8)$ & - & - \\
\hline \multicolumn{5}{|l|}{ Recruitment area } \\
\hline Thimphu & 1003 & $426(42.5)$ & Ref. cat. & \\
\hline Lungthenphu & 617 & $235(38.1)$ & 0.8 & $0.7-1.0$ \\
\hline \multicolumn{5}{|l|}{ Age groups (yrs) } \\
\hline $25-34$ & 852 & $407(47.8)$ & 2.3 & $1.8-3.0$ \\
\hline $35-44$ & 435 & $122(28.0)$ & Ref. cat. & \\
\hline$\geq 45$ & 333 & $132(39.6)$ & 1.7 & $1.2-2.3$ \\
\hline \multicolumn{5}{|l|}{ Occupation } \\
\hline Housewife & 1051 & $402(38.2)$ & Ref. cat. & \\
\hline Shopkeeper/saleswoman & 161 & $77(47.8)$ & 1.6 & $1.1-2.3$ \\
\hline Manual worker/Farmer & 154 & $81(52.6)$ & 1.7 & $1.2-2.4$ \\
\hline Clerical staff & 110 & $44(40.0)$ & 1.0 & $0.6-1.5$ \\
\hline Teacher/Health worker/Students & 131 & $48(36.7)$ & 1.0 & $0.6-1.4$ \\
\hline \multicolumn{5}{|l|}{ Ethnic group } \\
\hline Scharchop & 605 & $226(37.4)$ & Ref. cat. & \\
\hline Ngalop & 396 & $168(42.4)$ & 1.3 & $1.0-1.7$ \\
\hline Lhotsampa & 340 & $156(45.9)$ & 1.6 & $1.2-2.1$ \\
\hline Other & 274 & $106(38.7)$ & 1.1 & $0.8-1.5$ \\
\hline \multicolumn{5}{|l|}{ Marital status } \\
\hline Married & 1487 & $586(39.4)$ & Ref. cat. & \\
\hline Single & 15 & $12(80.0)$ & 4.9 & $1.3-17.9$ \\
\hline Widowed/separated & 112 & $57(50.9)$ & 1.8 & $1.2-2.6$ \\
\hline \multicolumn{5}{|l|}{ Pregnancies } \\
\hline 0 & 93 & $63(67.7)$ & 1.9 & $1.2-3.2$ \\
\hline 1 & 267 & $145(54.3)$ & Ref. cat. & \\
\hline 2 & 442 & $182(41.2)$ & 0.7 & $0.5-0.9$ \\
\hline$\geq 3$ & 809 & $263(32.5)$ & 0.6 & $0.4-0.8$ \\
\hline$x_{1}^{2}$ for trend & & & $=9.8, p=0.002^{b}$ & \\
\hline \multicolumn{5}{|l|}{ Contraceptive method } \\
\hline None & 343 & $194(56.6)$ & Ref. cat. & \\
\hline Hormonal & 656 & $261(39.8)$ & 0.5 & $0.3-0.6$ \\
\hline Other & 606 & $196(32.3)$ & 0.4 & $0.3-0.5$ \\
\hline \multicolumn{5}{|l|}{ Lifetime sexual partners } \\
\hline 1 & 1420 & $568(40.0)$ & Ref. cat. & \\
\hline$\geq 2$ & 174 & $77(44.2)$ & 1.4 & $1.0-1.9$ \\
\hline \multicolumn{5}{|l|}{ HPV infection } \\
\hline Negative & 1254 & $479(38.2)$ & Ref. cat. & \\
\hline Positive & 366 & $182(49.7)$ & 1.5 & $1.2-1.9$ \\
\hline
\end{tabular}

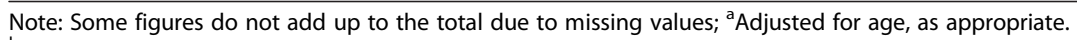

${ }^{\mathrm{b}}$ Trend assessed among ever pregnant women only. 

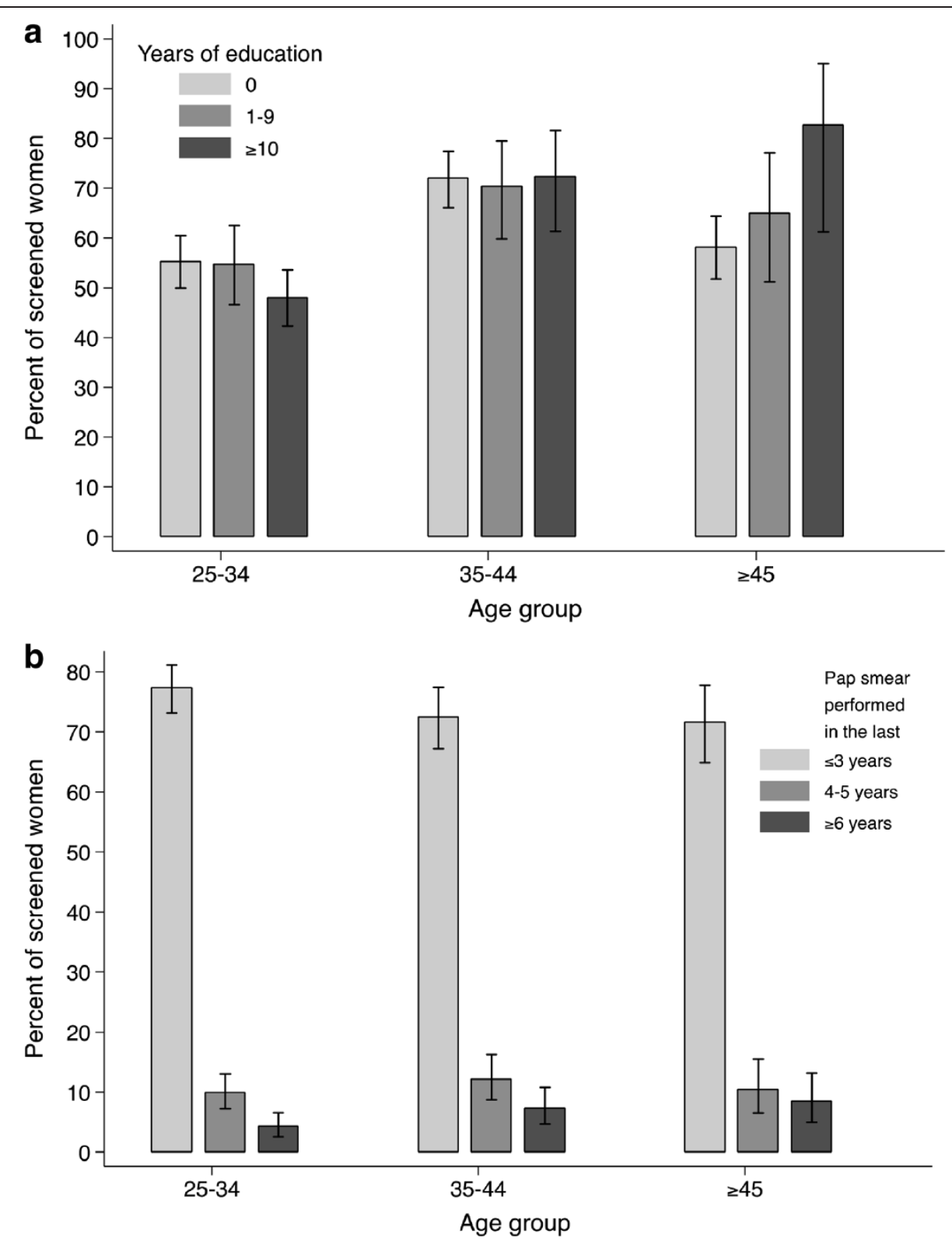

Figure 2 Percentage of screened women by age group and years of education, 1,620 women (a); or years elapsed since last Pap smear, 959 previously screened women (b); Thimphu and Lungthenphu, Bhutan.

screening attendance. Our findings provide, therefore, additional evidence $[21,22]$ on the limitations, in terms of population-based coverage, of mainly linking screening offer with attendance at perinatal care and family planning clinics.

Manual workers, farmers, shop-keepers and saleswomen reported previous screening less often than housewives but this was not the case for clerical staff, teachers and health workers. Economic reasons are unlikely to explain lack of screening in Bhutanese women because of the universal access to free health care. However, inconvenient opening hours at screening clinics and earning losses from attending may well discourage some women from being screened. Interestingly, education level was associated with screening attendance only among women aged 45 years or above. A larger fraction of never-screened women was also found in the Lhotsampa minority, a predominantly Hindu ethnic group in a predominantly Buddhist country. Finally, lack of previous screening was more frequent among women at higher cervical cancer risk, i.e., the few who reported two sexual partners or more and the many who were HPV-positive. 
Table 2 Odds ratio and $95 \%$ confidence interval for lack of prior screening by place invited and study participation, 1,503 women, Thimphu, Bhutan

\begin{tabular}{llllll}
\hline Place of invitation & Total & Ever screened N. (\%) & Never screened N. (\%) & Odds ratio $^{\text {a }}$ & 95\% confidence interval $^{\text {(1inic }}$ \\
\hline C78 & $457(58.7)$ & $321(41.3)$ & Ref. cat & $1.0-1.7$ \\
Home, participants & 225 & $120(53.3)$ & $105(46.7)$ & 1.3 & $3.4-5.6$ \\
Home, non-participants & 500 & $126(25.2)$ & $374(74.8)$ & 4.3 & $2.3-3.6$ \\
Home, all & 725 & $246(33.9)$ & $479(66.1)$ & 2.9 & \\
\hline
\end{tabular}

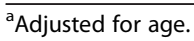

Cervical cancer screening is still not widely understood and accepted by Bhutanese women. A recent report [10] has explored knowledge on and attendance of cervical cancer screening in university graduated, and mainly unmarried, women (mean age 23 years). The study revealed that, despite national recommendations to start at age 20, approximately $95 \%$ of Bhutanese female university graduates had never been screened. The most frequently reported reasons for not attending screening were a) no awareness of the need for a Pap smear (57\%), b) embarrassment of being examined by male health professionals (24\%), and c) fear of being diagnosed with cancer (20\%). Similar reasons may explain lack of screening in a fraction of women in our present study.

Like in most low/middle-income countries and in all areas included in Figure 4, the population in the capital of Bhutan is rapidly growing, census is incomplete, and addresses inaccurate. The national screening program does not include an individual active call-and-recall system and women are invited to join the screening program through a variety of ways, e.g., mobilization at the community level, leaflets, posters, and messages on media. An accurate estimation of population-based screening coverage was, therefore, lacking. We have clearly shown that past screening attendance among women who accepted to join our study was affected by participation bias. Thanks to the information on screening history that was obtained during home visits we have been at least partially able to overcome this bias. Past screening attendance was 59\% among all participants but $34 \%$ in women contacted at home, and only $25 \%$ in women contacted at home who refused to participate in our study. Low screening attendance in women age 45 or older is especially concerning as it points to a large pool of undetected severe cancerous and precancerous cervical lesions in Thimphu [23]. Also of concern, less than one third of women invited from home joined our study.

Strengths of the present study include the large number and broad age range of participants, and the combination of different sampling methods that gave us the

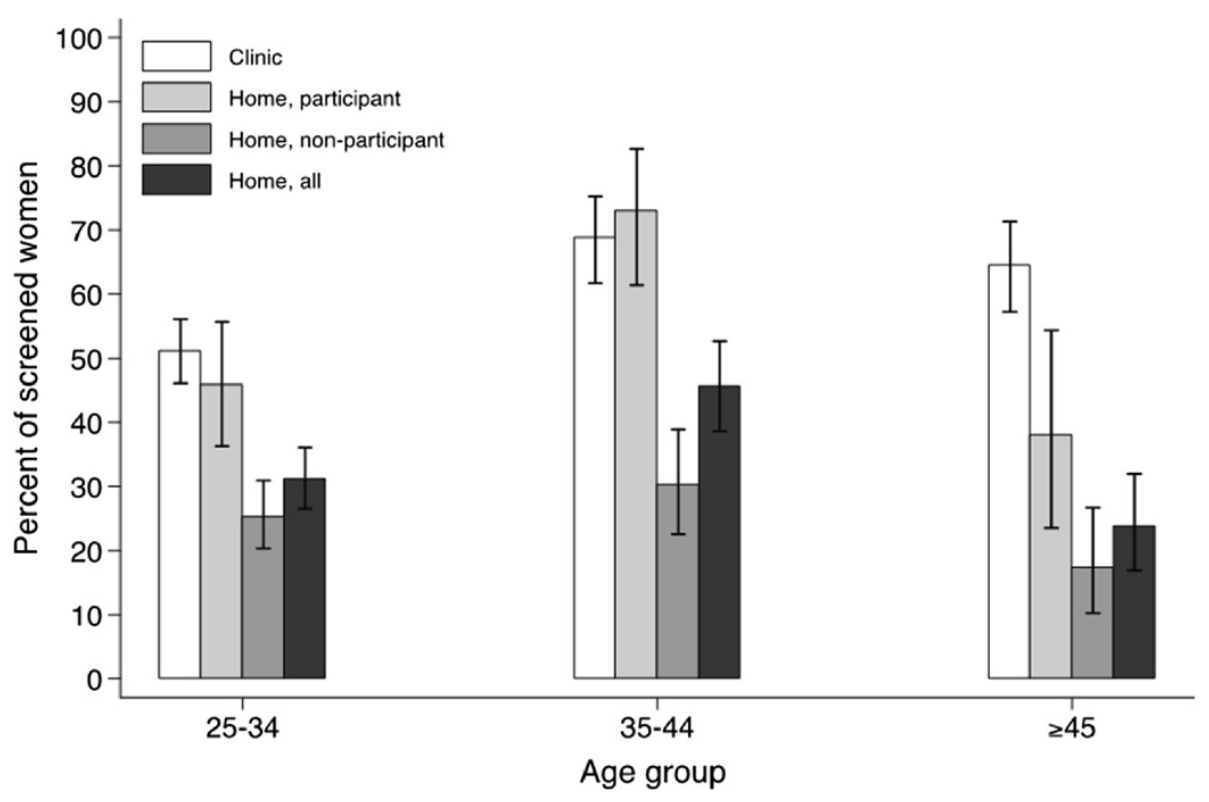

Figure 3 Percentage of screened women by place contacted, study participation, and age group, 1,503 women, Thimphu, Bhutan. 
possibility to tackle participation bias. Some control of self-reported screening history could be made among women who attended study clinics by crosschecking women's answers with the records of JDWNRH, Department of Laboratory Services, the only cytology provider in Thimphu. Screening history collected at home among non-participants may have been less accurate than information obtained during study interview for participants, as some women may not have fully understood the question. Obviously, we have no information on screening attendance among women who were never found at home by social workers, despite repeated attempts.

\section{Conclusions}

The transition from an opportunistic screening to an allreaching population-based screening is yet to be achieved in the capital of Bhutan, let alone in the entire country. An active individual call and recall system has been a key factor in improving screening coverage in western countries [22] but invitation at home did not work very well in never-screened women in Thimphu.

A wider offer of screening also outside maternal and child health clinics, and better health education are needed. Ad hoc methods to target never-screened women, e.g., through campaigns set in working places, markets, religious events, and other public meeting places, are however the priority in Bhutan. As shown in the Netherlands
[24], self-collection of vaginal samples in combination with HPV testing may overcome women's reluctance to attend a gynaecological examination in Thimphu and also facilitate the performance of cervical cancer screening in rural and high-altitude areas in Bhutan.

\section{Abbreviations}

Cl: Confidence interval; HPV: Human papillomavirus; IARC: International Agency for Research on Cancer; ICC: Invasive cervical cancer; JDWNRH: Jigme Dorji Wangchuck National Referral Hospital; OR: Odds ratio.

\section{Competing interests}

The authors declare that they have no competing interests.

\section{Authors' contributions}

IB contributed to study design and implementation, data analysis, and manuscript preparation. UT contributed to study design and

implementation, and manuscript preparation. VT contributed to study implementation and data analysis. TT contributed to study implementation and manuscript preparation. SF and GMC conceived the study, contributed to study design and implementation, data analysis, and manuscript preparation. All authors read and approved the final manuscript.

\section{Acknowledgements}

Primary support for this project came from the Bill \& Melinda Gates Foundation, USA (grant numbers 35537 and OPP1053353).

We would like to thank the women who participated and the contribution of the following staff at JDWNRH, Thimphu and Lungthenphu Hospital. In particular, we acknowledge the contribution from Drs C Zangmo

(colposcopical examination), IK Mohanta and BM Dhungel (cytopathology), and Mr K Wangchuk and Ms Samten (data entry). We also thank Drs PJF Snijders, and CJLM Meijer, VU University Medical Center, Amsterdam, The Netherlands, for performing HPV testing. Ms S Gamon provided skilled technical assistance. 


\section{Author details}

'International Agency for Research on Cancer, 150 cours Albert Thomas, 69372 Lyon Cedex 08, France. ${ }^{2}$ Department of Obstetrics \& Gynaecology, Jigme Dorji Wangchuck National Referral Hospital, Thimphu, Bhutan. ${ }^{3}$ Department of Laboratory Services, Jigme Dorji Wangchuck National Referral Hospital, Thimphu, Bhutan.

Received: 25 June 2014 Accepted: 10 November 2014

Published online: 30 November 2014

\section{References}

1. Ferlay J, Soerjomataram I, Ervik M, Dikshit R, Eser S, Mathers C, Rebelo M, Parkin DM, Forman D, Bray F: GLOBOCAN 2012 v1.0, Cancer Incidence and Mortality Worlwide: IARC CancerBase No. 11 [Internet]. Lyon, France: International Agency for Research on Cancer; 2013.

2. Tshomo U, Franceschi S, Dorji D, Baussano I, Tenet V, Snijders PJ, Meijer CJ, Bleeker MC, Gheit T, Tommasino M, Clifford GM: Human papillomavirus infection in Bhutan at the moment of implementation of a national HPV vaccination programme. BMC Infect Dis 2014, 14:408.

3. Sukvirach S, Smith JS, Tunsakul S, Munoz N, Kesararat V, Opasatian O, Chichareon S, Kaenploy V, Ashley R, Meijer CJ, Snijders PJ, Coursaget P, Franceschi S, Herrero R: Population-based human papillomavirus prevalence in Lampang and Songkla, Thailand. J Infect Dis 2003, 187:1246-1256.

4. Anh PT, Hieu NT, Herrero R, Vaccarella S, Smith JS, Thuy NT, Nga NH, Duc NB, Ashley R, Snijders PJ, Meijer CJ, Munoz N, Parkin DM, Franceschi S: Human papillomavirus infection among women in South and North Vietnam. Int J Cancer 2003, 104:213-220.

5. Li LK, Dai M, Clifford GM, Yao WQ, Arslan A, Li N, Shi JF, Snijders PJ, Meijer CJ, Qiao YL, Franceschi S: Human papillomavirus infection in Shenyang City, People's Republic of China: a population-based study. Br J Cancer 2006, 95:1593-1597.

6. Dai M, Bao YP, Li N, Clifford GM, Vaccarella S, Snijders PJF, Huang RD, Sun LX, Meijer CJLM, Qiao YL, Franceschi S: Human papillomavirus infection in Shanxi Province, People's Republic of China: a population-based study. Br J Cancer 2006, 95:96-101.

7. Wu RF, Dai M, Qiao YL, Clifford GM, Liu ZH, Arslan A, Li N, Shi JF, Snijders PJ, Meijer CJ, Franceschi S: Human papillomavirus infection in women in Shenzhen City, People's Republic of China, a population typical of recent Chinese urbanisation. Int J Cancer 2007, 121:1306-1311.

8. Dondog B, Clifford GM, Vaccarella S, Waterboer T, Unurjargal D, Avirmed D, Enkhtuya S, Kommoss F, Wentzensen N, Snijders PJ, Meijer CJ, Franceschi S, Pawlita M: Human papillomavirus infection in Ulaanbaatar, Mongolia: a population-based study. Cancer Epidemiol Biomarkers Prev 2008, 17:1731-1738.

9. Sherpa AT, Clifford GM, Vaccarella S, Shrestha S, Nygård M, Karki BS, Snijders PJ, Meijer CJ, Franceschi S: Human papillomavirus infection in women with and without cervical cancer in Nepal. Cancer Causes Control 2010, 21:323-330.

10. Dhendup T, Tshering P: Cervical cancer knowledge and screening behaviors among female university graduates of year 2012 attending national graduate orientation program, Bhutan. BMC Womens Health 2014, 14:44.

11. Dorji T, Tshomo U: Early Implementation and Monitoring of HPV Vaccination in Bhutan. In World Cancer Report 2014. Edited by Stewart BW, Wild CP. Lyon: International Agency for Research on Cancer; 2014:317.

12. van den Brule AJ, Pol R, Fransen-Daalmeijer N, Schouls LM, Meijer CJ, Snijders PJ: GP5+/6+ PCR followed by reverse line blot analysis enables rapid and high-throughput identification of human papillomavirus genotypes. J Clin Microbiol 2002, 40:779-787.

13. Sasieni $P$, Adams J, Cuzick J: Benefit of cervical screening at different ages: evidence from the UK audit of screening histories. Br J Cancer 2003, 89:88-93.

14. Matos E, Loria D, Amestoy G, Herrera L, Prince MA, Moreno J, Krunfly C, van den Brule AJ, Meijer CJ, Munoz N, Herrero R, Proyecto Concordia Collaborative Group: Prevalence of human papillomavirus infection among women in Concordia, Argentina: a population-based study. Sex Transm Dis 2003, 30:593-599.

15. Thomas JO, Herrero R, Omigbodun AA, Ojemakinde K, Ajayi IO, Fawole A, Oladepo O, Smith JS, Arslan A, Munoz N, Snijders PJ, Meijer CJ, Franceschi S: Prevalence of papillomavirus infection in women in Ibadan, Nigeria: a population-based study. Br J Cancer 2004, 90:638-645.
16. Keita N, Clifford GM, Koulibaly M, Douno K, Kabba I, Haba M, Sylla BS, van Kemenade FJ, Snijders PJ, Meijer CJ, Franceschi S: HPV infection in women with and without cervical cancer in Conakry, Guinea. Br J Cancer 2009, 101:202-208.

17. Hammouda D, Clifford GM, Pallardy S, Ayyach G, Chekiri A, Boudrich A, Snijders PJ, van Kemenade FJ, Meijer CJ, Bouhadef A, Zitouni Z, Habib D, Ikezaren N, Franceschi S: Human papillomavirus infection in a population-based sample of women in Algiers, Algeria. Int J Cancer 2011, 128:2224-2229.

18. Raza SA, Franceschi S, Pallardy S, Malik FR, Avan Bl, Zafar A, Ali SH, Pervez S, Serajuddaula S, Snijders PJ, van Kemenade FJ, Meijer CJ, Shershah S, Clifford GM: Human papillomavirus infection in women with and without cervical cancer in Karachi, Pakistan. Br J Cancer 2010, 102:1657-1660.

19. Alibegashvili T, Clifford GM, Vaccarella S, Baidoshvili A, Gogiashvili L, Tsagareli Z, Kureli I, Snijders PJ, Heideman DA, van Kemenade FJ, Meijer CJ, Kordzaia D, Franceschi S: Human papillomavirus infection in women with and without cervical cancer in Tbilisi, Georgia. Cancer Epidemiol 2011, 35:465-470

20. Khodakarami N, Clifford GM, Yavari P, Farzaneh F, Salehpour S, Broutet N, Bathija H, Heideman DA, van Kemenade FJ, Meijer CJ, Hosseini SJ, Franceschi S: Human papillomavirus infection in women with and without cervical cancer in Tehran, Iran. Int J Cancer 2012, 131:E156-E161.

21. Sankaranarayanan R, Budukh AM, Rajkumar R: Effective screening programmes for cervical cancer in low- and middle-income developing countries. Bull World Health Organ 2001, 79:954-962.

22. IARC: IARC Handbooks of Cancer Prevention Volume 10: Cervix Cancer Screening. Lyon, France: IARC Press; 2005.

23. Schiffman M, Castle PE, Jeronimo J, Rodriguez AC, Wacholder S: Human papillomavirus and cervical cancer. Lancet 2007, 370:890-907.

24. Verhoef VM, Bosgraaf RP, van Kemenade FJ, Rozendaal L, Heideman DA, Hesselink AT, Bekkers RL, Steenbergen RD, Massuger LF, Melchers WJ, Bulten J, Overbeek LI, Berkhof J, Snijders PJ, Meijer CJ: Triage by methylation-marker testing versus cytology in women who test HPV-positive on self-collected cervicovaginal specimens (PROHTECT-3): a randomised controlled non-inferiority trial. Lancet Oncol 2014, 15:315-322.

doi:10.1186/s12905-014-0147-0

Cite this article as: Baussano et al: Cervical cancer screening program in Thimphu, Bhutan: population coverage and characteristics associated with screening attendance. BMC Women's Health 2014 14:147.

\section{Submit your next manuscript to BioMed Central and take full advantage of:}

- Convenient online submission

- Thorough peer review

- No space constraints or color figure charges

- Immediate publication on acceptance

- Inclusion in PubMed, CAS, Scopus and Google Scholar

- Research which is freely available for redistribution 